\title{
Buckling and Vibration ANALysis of LAMinated Panels Using VICONOPT
}

\author{
By David Kennedy, ${ }^{1}$ Fred W. Williams, ${ }^{2}$ and Melvin S. Anderson ${ }^{3}$
}

\begin{abstract}
The analysis aspects of the 23,000 -line FORTRAN program VICONOPT are described. Overall stiffness matrices assembled from the earlier exact VIPASA flat plate stiffnesses are optionally coupled by Lagrangian multipliers to find critical buckling loads, or natural frequencies of undamped vibration, of prismatic assemblies of anisotropic flat plates with arbitrarily located point supports or simple transverse supporting frames. The longitudinal continuity of typical wing and fuselage panels is closely approximated because the solutions are for the infinitely long structure obtained by repeating a bay and its supports longitudinally. Any longitudinally invariant in-plane plate stresses are permitted, and very rapid solutions are guaranteed by numerous refinements, including multilevel substructuring and a method for repetitive cross sections that is exact for regular polygons used to represent cylinders. Modal displacements and stresses in or between plies of laminated plates are calculated and plotted, with values being recovered at all nodes of substructures. Comparison with usual approximate finite-element methods confirms that, for comparably converged solutions, VICONOPT is typically between 100 and $10^{4}$ times faster.
\end{abstract}

\section{INTRODUCTION}

The FORTRAN 77 program VICONOPT (VIPASA with constraints and optimization) has approximately 23,000 lines and incorporates the earlier VIPASA (Vibration and instability of plate assemblies including shear and anisotropy) (Wittrick and Williams 1974) and VICON (VIPASA with constraints) (Anderson et al. 1983). It covers any prismatic assembly of anisotropic plates, and Fig. 1(a) shows typical cross sections. Each plate can carry any combination of $N_{L}, N_{T}$, and $N_{S}$, the longitudinally invariant inplane forces per unit length of plate edge shown in Fig. 1(b), where negative $N_{L}$ and $N_{T}$ give tension. Analysis or optimum design can be performed. Design aspects are described elsewhere (Butler and Williams 1990). The present paper covers the analysis features; these concern the calculation of eigenvalues, i.e. critical buckling load factors or undamped natural frequencies, the corresponding mode shapes, and the perturbation stress levels caused by these modes.

Development of VIPASA was led by Fred W. Williams and the late W. H. Wittrick at the University of Birmingham (Wittrick and Williams 1974; Williams and Anderson 1973). After enhancement by Anderson et al. (1976) at NASA-Langley Research Center, VIPASA was released by COSMIC. The theory and a preliminary version of VICON were developed at the University of Wales Institute of Science and Technology (UWIST) in collaboration with NASA and British Aerospace (Anderson et al. 1983).

\footnotetext{
${ }^{1}$ Sr. Res. Assoc., School of Engrg., Univ. of Wales Coll. of Cardiff, Cardiff, Wales, United Kingdom.

${ }^{2}$ Prof., Head of Struct. Div., School of Engrg., Univ. of Wales Coll. of Cardiff, Cardiff, Wales, United Kingdom.

${ }^{3}$ Res. Prof., Old Dominion Univ., Norfolk, Va.

Note. Discussion open until December 1, 1994. To extend the closing date one month, a written request must be filed with the ASCE Manager of Journals. The manuscript for this paper was submitted for review and possible publication on April 9, 1991. This paper is part of the Journal of Aerospace Engineering, Vol. 7, No. 3, July, 1994. CASCE, ISSN 0893-1321/94/0003-0245/ $\$ 2.00+\$ .25$ per page. Paper No. 1746.
} 


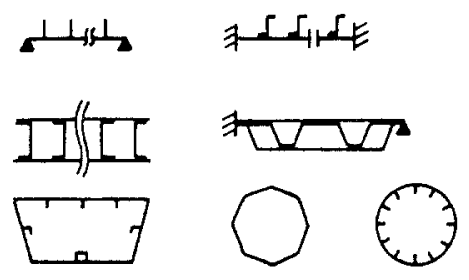

(a)

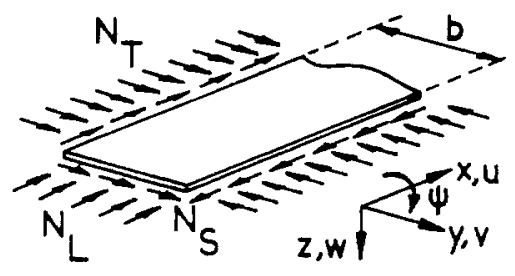

(b)

FIG. 1. (a) Cross Sections of Typical Prismatic Plate Assemblies Covered; (b) Component Plate, Showing Basic Force System, Plate Axis System, and Associated Displacement Amplitudes

(UWIST has since merged to become the University of Wales College of Cardiff.) Similar collaboration led to VICONOPT (Williams et al. 1990a), which is much more user friendly, has additional analysis features, has a design capability, and has been released to U.S. users by COSMIC. The present paper describes its analysis capabilities sufficiently for engineers to assess its relevance. Many of its novel theoretical aspects have already been published and are cross referenced, although some are described for the first time here and in a related conference paper (Williams et al. 1990b). Example problems were chosen to illustrate the scope of the program and solution times and storage requirements are given to enable its performance to be compared with competing programs. The basis is also given for formulas that the program uses to obtain a preliminary prediction of solution times, thus enabling users to plan computer runs accordingly.

\section{SUMMARY OF VIPASA THEORY}

VIPASA (Wittrick and Williams 1974) uses the stiffness matrix method based on exact flat plate theory, with Winkler foundations recently added (Kennedy and Williams 1990). The buckling or vibration mode is assumed to vary sinusoidally in the longitudinal direction $x$, wit half-wavelength $\lambda$ and with the displacement amplitudes $u, v, w$, and $\psi$ shown in Fig. $1(b)$. Computations are performed separately for each $\lambda$ specified by the user. It also uses an algorithm (Wittrick and Williams 1971, 1973; Williams and Wittrick 1983) that guarantees convergence on all required eigenvalues and permits very concise and flexible use of multilevel substructuring (Williams 1972) to reduce computation, data preparation, and computer memory usage.

Plate bending and membrane behaviors are assumed to be uncoupled and orthotropy is assumed for the membrane case with the principal elastic axes parallel to the $x$ - and $y$-axes of Fig. 1(b). Thus, using the usual symbols, the matrices $\mathbf{B}, \mathbf{D}$, and $\mathbf{A}$ are, respectively, null, fully populated, and such that $A_{13}=A_{23}=0$. Hence the commonly used balanced symmetric laminates are included. The "nodal" lines of zero displacement are straight and in the $y$-direction of Fig. 1(b) if all plates are either isotropic or orthotropic (i.e. having $D_{13}=D_{23}=0$ ) with $N_{S}=0$ and so satisfy transverse simply supported end conditions provided that $\lambda$ divides exactly into the length $l$. This case gives the fastest execution times because all the stiffness calculations use real arithmetic. Otherwise (i.e. if any plates are anisotropic or have $N_{S} \neq 0$ ), solutions only approach such end conditions, and when $N_{S}$ is large they become excessively conservative as $\lambda$ approaches $l$. This case 
results in the stiffness matrix of the structure being complex (Wittrick and Williams 1974), although real arithmetic is still used for any substructures in which each plate is isotropic or orthotropic with $N_{S}=0$.

Dead load values of $N_{L}, N_{T}$, and $N_{S}$ are permitted for both buckling and vibration problems. In the former case they are additional to live load values which are factored until buckling occurs. Plate loadings may be given as data, although the program usually calculates $N_{L}$ from the total longitudinal load on the panel or from a uniform longitudinal strain, optionally allowing for temperature changes between plies in a laminated plate.

\section{SUMMARY OF VICON ENHANCEMENTS TO VIPASA}

VICON overcomes the difficulty that VIPASA solutions do not always approximate simply supported end conditions adequately when plates are anisotropic or have $N_{S} \neq 0$, by using Lagrangian multipliers (Anderson et al. 1983) to minimize the total energy of the panel (including inertia effects for vibration cases) subject to constraints that represent rigid or elastic point supports. Hence a shear loaded panel supported along rectangular boundaries can be represented to high accuracy, as in Fig. 2, which shows a polygonal blade stiffened panel with point supports (denoted by crosses) used to approximate transverse line supports. Alternatively, Fig. 2 shows that the constraints can be point attachments (denoted by circles) to supporting structures comprising straight beam-columns that lie in the $y-z$ plane, and that repeat at longitudinal intervals of $l$. The solution is a Fourier series involving appropriate half-wavelengths $\lambda$. Thus results are for an infinitely long plate assembly, with supports repeating at intervals of $l$. This accounts for the continuity over several bays of typical aerospace construction. A panel of finite length $l$ with simply supported ends may be modeled reasonably accurately by representing the simple supports by a line of rigid point supports at $x=0$. The results assume that the mode repeats over a length $L=2 l / \xi$ for some value $0 \leq \xi \leq 1$. Each of the values of $\xi$ chosen by the user generates an infinite series of $\lambda$ (Anderson et al. 1983), although a small finite number usually gives acceptable results, and it is necessary to find the $\xi$ that gives the lowest answer. For $\xi=0$ the mode repeats at intervals of $l$ and so is the same for all longitudinal bays.

This approach is necessarily slower than a VIPASA analysis involving a single value of $\lambda$. Therefore VIPASA analysis is recommended for the majority of cases, because it gives exact results when anisotropy, shear and

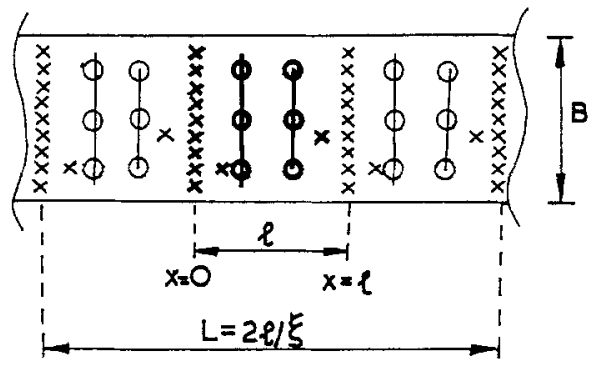

(a)

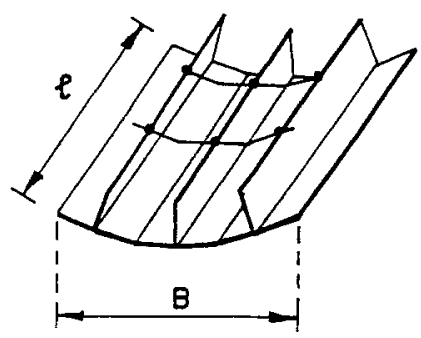

(b)

FIG. 2. Infinitely Long Structure: (a) Plan View with $\xi=2 / 3$; (b) Isometric View 
constraints are absent and otherwise usually gives acceptable accuracy, unless $\lambda$ approaches $l$ for panels with large values of $N_{S}$ or unless point supports are used for purposes other than representing transverse simply supported ends.

Plate assemblies that repeat in the global $y$-direction are analyzed by using infinite width recurrence relations (Williams and Anderson 1985) so that analysis involves only a datum-repeating portion. Such a structure is illustrated in Fig. 3. When used with the cylindrical coordinate option outlined later, this rapid analysis gives the same result for regular polygonal cross sections, including stiffened or corrugated ones, as would be given by modeling the compete polygon.

The "infinitely wide" analysis is analogous to the "infinitely long" analysis of Fig. 2. To approximate a panel comprising $P$ repeating portions each of width $B$ [see Fig. 3(a)] that is simply supported at its longitudinal edges, only modes that repeat at transverse intervals of $2 P B$ should be analyzed, by considering only transverse half-wavelengths $\lambda_{T}=B / \eta$ for $\eta=0, \pm 1 /$ $P, \pm 2 / P, \ldots,+1$. This condition gives exact results in VIPASA analyses when shear and anisotropy are absent, and also in certain VICON analyses when the nodal lines run longitudinally. For $\eta=0$, all repeating portions deflect identically. [Negative values of $\eta$ are required because of the complex arithmetic formulation used; see Williams and Anderson (1985).]

\section{FURTHER DETAILS OF ANALYSIS FEATURES}

The two previous sections showed that finding the lowest eigenvalue of a plate assembly involves repeating the analysis for several values of $\lambda$ and/or $\xi$, and also of $\eta$ for a repetitive structure. The FAST option accelerates this process by omitting unnecessary calculation of eigenvalues which exceed the lowest one found already, as follows.

The program finds eigenvalues by performing iterations, at each of which the eigenparameter is changed and the algorithm (Wittrick and Williams $1971,1973)$ checks how many eigenvalues have been exceeded. For the second and subsequent combinations of $\lambda$ or $\xi$ and $\eta$, the first iteration is at the lowest eigenvalue found already and no further iterations are performed if no eigenvalues have been exceeded there. This method saves

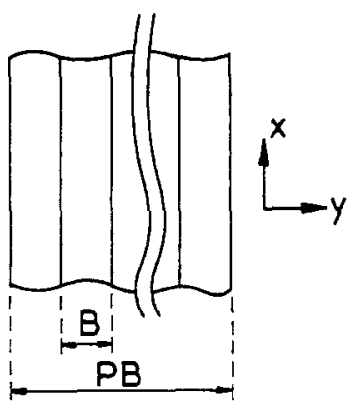

(a)

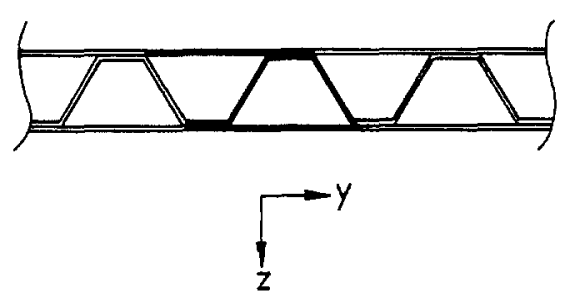

(b)

FIG. 3. Repetitive Plate Assemblies: (a) Plan View, Showing P Repeating Portions; (b) Typical Cross Section, with Datum-Repeating Portion Shown Bold 
much time if the first combination of values of $\lambda$ or $\xi$ and $\eta$ chosen is that which gives the lowest eigenvalue. The user is allowed to choose the order of the various combinations, so that those most likely to yield the lowest buckling load are tried first. If VIPASA and VICON analyses are selected in the same run, the faster VIPASA analysis is performed first to save time.

VICONOPT can analyze in a single run several variants of the basic problem, e.g. multiple load cases, by means of very concise extra data. Then the FAST option can accelerate the analysis if the only eigenvalue required is the overall lowest for the basic problem and its variants.

The data permits concise definition of individual isotropic, anisotropic or laminated plates. Fig. 4 shows how these may be aligned by rotation, by applying offsets at edges or by a cylindrical coordinate transformation which simplifies the modeling of polygonal cross sections. Such aligned plates are connected together rigidly and may be formed into substructures before being inserted in the final assembly. The substructures must form chains, i.e. each node can only be connected to the nodes immediately preceding and following it by a plate or any previously defined substructure. Singly connected substructures may be formed by attaching one edge of a plate or previously defined substructure to any node of a substructure chain or to a node of the final assembly. Because substructures can be nested to any level modeling is very adaptable, so that nearly all practical structures can be modeled efficiently. Previous examples (Williams 1972) illustrate this, as

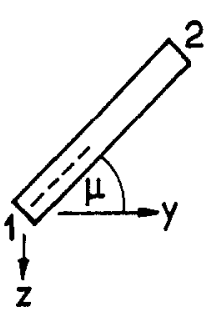

(a)

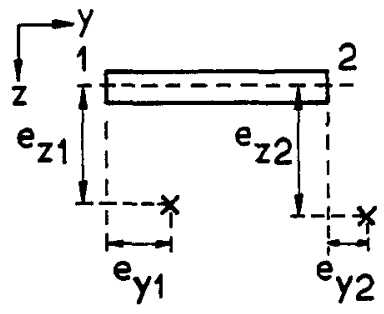

(b)

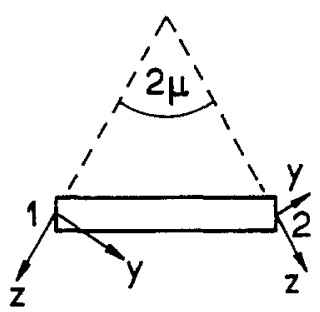

(c)

FIG. 4. Plate Alignment Options: (a) Rotation; (b) Offsets; (c) Cylindrical Coordinates

(a)

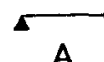

A

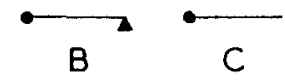

$C$

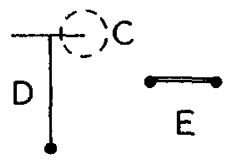

$E$

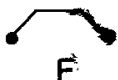

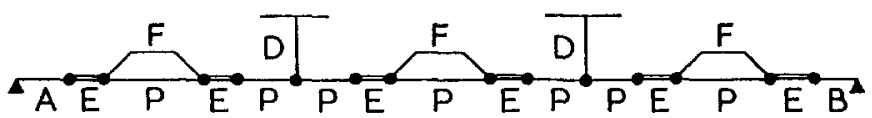

FIG. 5. Assembly by Substructuring: (a) Substructures A-F; (b) Parent Cross Section, Showing Nodes ( $\bullet$ ), Longitudinal Supports (A), and Individual Plates (P) 
does Fig. 5, on which the longitudinal supports are rigid or are longitudinally invariant elastic line supports and for which the User Manual (Williams et al. 1990a) gives full data preparation details. Here substructures are singly $(A-D)$ or doubly ( $E$ and $F$ ) connected. Note that $D$ requires prior assembly of $\mathrm{C}$ to make its nodes form a chain, so that two levels of substructuring are required. The use of $\mathrm{F}$ in Fig. $5(b)$ illustrates the usefulness of multiple connections (two here) between the same pair of nodes. Similarly, several singly connected substructures can be connected to the same parent structure node. The substructures $D$ and $F$ were introduced to reduce data preparation and computer run times, both because they need be defined and analyzed only once, and also because they make the nodes of Fig. 5(b) form a chain, thus minimizing the bandwidth of the associated stiffness matrix.

The operations of Fig. 4 are applicable to substructures and can be applied in any order to the same plate or substructure (Williams et al. 1990a). Transforming into and out of cylindrical coordinates at appropriate stages can be very efficient when using substructures to model cross sections that involve several regular polygonal portions, e.g. to represent cylindrical portions of differing radius. The theory for transformation to cylindrical coordinates is given elsewhere (Williams and Wright 1978) and the plate alignment and substructuring theory used was given when VIPASA was written (Wittrick and Williams 1974).

Laminated walls can be assembled from any sequence of arbitrarily oriented orthotropic plies. In general $A_{13}$ and $A_{23}$ are nonzero for unbalanced laminates, while the $\mathbf{B}$ matrix exists for unsymmetric laminates. The first of these effects is ignored. The second is partially eliminated by a procedure that automatically reduces terms in $\mathbf{B}$ (which are then ignored) while adjusting terms in $\mathbf{D}$, by redefining the reference surface away from the midplane of the wall and then automatically applying the offset transformation of Fig. $4(b)$. Alternatively, when balanced symmetric laminates are bonded to form an unsymmetric whole (e.g. E on Fig. 5), the laminates can be divided into narrow strips that are then connected together by offset connections.

VIPASA and VICON mode finding routines calculated only the deflections at the nodes of the final structure and then interpolated modes between them. The perturbation stresses caused by these modes were not calculated. VICONOPT additionally permits calculation of the displacements of all internal nodes of substructures and of the resulting perturbation stresses including, for laminated plates, the in-plane direct and shear stresses in plies and the interply shear stresses. The program has many plot options, including contour plots of any of these stresses for selected plates and contour, isometric, or cross section plots of deflections. Undeflected cross section plots show individual plies of laminated plates. These features are easily portable between computers, and are largely due to a colleague, Dr. G. Aston, of British Aerospace. Although they involved no new fundamental theory, they needed extensive logical thinking and about 2,500 lines of very involved coding. This is because they had to mesh with the alignment and substructuring features just discussed; see Figs. 4 and 5 . Particular difficulties were that the number of levels of substructuring is unlimited and that a given type of substructure appears only once in data and in computations (prior to mode finding) but can appear often in the structure, possibly within parent substructures that are at different substructure levels. Stresses within a plate may optionally be found by making an automatic, temporary division into a user chosen number of narrower plates 
and using the substructure method just described to calculate the displacements, and hence the stresses, at their longitudinal edges.

\section{RUN TIMES}

The lengthy derivation of (1) and (2) is given elsewhere [Anderson et al. (1983), where these equations are numbered (A1) and (32) and use a slightly different notation].

For the present purpose this application of the algorithm to the Lagrangian multiplier method of VICON can be summarized as follows. The number of eigenvalues exceeded by a trial value of the eigenparameter, $J$, is given by

$$
J=\alpha_{f}\left(J_{0 f}+s\left\{\mathbf{K}_{f}\right\}+s\{\mathbf{R}\}-r\right)+\sum \alpha_{i}\left(J_{0 i}+s\left\{\mathbf{K}_{i}\right\}\right)
$$

where

$$
\mathbf{R}=-\mathbf{e}_{f} \mathbf{K}_{f}^{-1} \mathbf{e}_{f}^{T}-\frac{1}{l} \sum \mathbf{e}_{i} \mathbf{K}_{i}^{-1} \mathbf{e}_{i}^{H}
$$

Here $\mathbf{K}_{f}=$ stiffness matrix of any supporting structure; the $\mathbf{K}_{i}=$ VIPASA stiffness matrices for the $\lambda$ s coupled by the Lagrangian multipliers and the summations are over these $\lambda \mathrm{s}$; and $r=$ number of constraints imposed by the Lagrangian multipliers over length $l$ [e.g. 17 for Fig. 2(a) if each cross or circle constrains only one degree of freedom]. The $\mathbf{e}_{i}, \mathbf{e}_{f}$, and $\mathbf{R}$ (which is $r \times r)$ are generated by these constraints. Superscripts $T$ and $H$ denote transpose and Hermitian transpose; and $\alpha_{f}$ and the $\alpha_{i}$ are all equal to unity or two, depending upon the problem. The $J_{0 i}$ and $J_{0 f}$ are the contributions that would be made to $J$ if all nodes of the final structure and supporting structure were fully clamped, and they are calculated by methods described previously (Anderson et al. 1983). $s\{\}$ denotes the sign count (Wittrick and Williams 1971) of the matrix within the curly braces; i.e. such that $s\left\{\mathbf{K}_{i}\right\}$ equals the number of negative leading diagonal elements of $\mathbf{K}_{i}^{\Delta}$, the upper triangular form of $\mathbf{K}_{i}$ obtained by applying Gauss elimination in the form in which rows are taken as pivotal in order and multiples of the pivotal row are added to unscaled succeeding rows. For any given problem the $\mathbf{K}_{i}$ have order $f N \times f N$ and bandwidth $f M$, where $N$ is the number of nodes of the final structure, $M$ exceeds by one the maximum node number difference for any pair of connected nodes and $f$ is the number of degrees of freedom at each node. Here $f=4$ to account for the four displacement amplitudes at each node shown in Fig. 1(b), but $f=3$ for the plane frame supporting structures described later. From (2), $\mathbf{e}_{i}$ is $r \times f N$ and $\mathbf{e}_{f}$ has $r$ rows and as many columns as $\mathbf{K}_{f}$.

VICONOPT calculates $\mathbf{e}_{i} \mathbf{K}_{i}^{-1} \mathbf{e}_{i}^{H}$ in (2) and the $s\left\{\mathbf{K}_{i}\right\}$ in (1) by a single standard substructuring operation, in which $f=4$ and the $(f N+r) \times(f N$ $+r$ ) Hermitian matrix

$$
\left[\begin{array}{cc}
\mathbf{K}_{i} & \mathbf{e}_{i}^{H} \\
\mathbf{e}_{i} & \mathbf{0}
\end{array}\right]
$$

has Gauss elimination applied to it until $f N$ rows have been pivotal. Hence $\mathbf{K}_{i}^{\Delta}$ replaces $\mathbf{K}_{i}$ and $-\mathbf{e}_{i} \mathbf{K}_{i}^{-1} \mathbf{e}_{i}^{H}$ replaces the null matrix. Applying standard techniques, it is easily demonstrated that this involves

$$
t_{1} \simeq \frac{1}{2}\left\{f^{3}\left(N-\frac{2}{3} M\right) M^{2}+f^{2}(N-M) M r+\frac{1}{3} f N r^{2}\right\}
$$


multiplications. This expression is equivalent to that given earlier (Williams and Anderson 1985), but has been modified to allow for savings when the last $f M$ rows of $\mathbf{K}_{i}$ are pivotal.

A similar procedure gives $\mathbf{e}_{f} \mathbf{K}_{f}^{-1} \mathbf{e}_{f}^{T}$ and $s\left\{\mathbf{K}_{f}\right\}$, except that, since supporting structures consist of an uncoupled set of transverse plane frames that are perpendicular to the $x$-axis, computer time is saved by treating each frame separately. Therefore the formula for $t_{2}$, the number of multiplications needed, is identical to (3) except that $N$ and $M$ are replaced by the corresponding values for the frame, $f=3$ and summation over all supporting frames is required. The number of multiplications needed to compute $s\{\mathbf{R}\}$ in (1) is

$$
t_{3} \simeq \frac{1}{6} r^{3}
$$

Substructuring is particularly useful when using VICON analysis because it reduces $N$ and hence also $t_{1}$, e.g. see (3) and substructures $\mathrm{A}$ and $\mathrm{B}$ in Fig. 5. However, substructures cannot include constraints, which restricts their use. The program sets up the $f N_{s} \times f N_{s}$ stiffness matrix corresponding to the $N_{s}$ nodes of a substructure, with its $\left(M_{s}-1\right)$ connection nodes numbered last. The bandwidth is $f M_{s}$, where $M_{s}$ is 2 for singly ( 3 for doubly) connected substructures and again $f=4$. Analogously to the computation of $t_{1}$ in (3), Gauss elimination is applied until all except the last $f\left(M_{s}-1\right)$ rows have been pivotal. This involves

$$
t_{4} \simeq \frac{1}{2} \sum f^{3}\left(N_{s}-\frac{2}{3} M_{s}\right) M_{s}^{2}
$$

multiplications, where the summation is over all substructures.

Substructuring can also be used when assembling supporting structures, to reduce the order of $\mathbf{K}_{f}$, and hence to reduce $t_{2}$. This involves $t_{5}$ multiplications, where the formula for $t_{5}$ duplicates (5), with $N_{s}$ and $M_{s}$ now being for the frame substructures and with $f=3$. Such substructures, and their component Timoshenko beam-column members, can be aligned just as plates can; see Fig. 4. Hence they can be singly or doubly connected and can be used in the multilevel way illustrated by Fig. 5 .

Computing plate and beam stiffnesses may take significant time. Therefore when there are $t_{6}$ plate types and $t_{7}$ beam types the program estimates the VICON type iteration time by multiplying each of $t_{1}, \ldots, t_{7}$ by appropriate constants and then summing. The constants clearly depend upon the computer, the number of $\mathbf{K}_{i}$ used, and the extent to which complex arithmetic is needed. The User Manual (Williams et al. 1990a) gives the detailed formulas and the corresponding formulas for VIPASA analysis (which use $r=t_{2}=t_{5}=t_{7}=0$ ) and for the transversely repetitive structure method described around Fig. 3. The computer-dependent constants used in these formulas must be found by using measured computer times for typical problems. Thus their values are automatically inflated to compensate for all operations (e.g. those of Fig. 4) that are omitted by $t_{1} \ldots, t_{7}$.

The program does not estimate the number of iterations required. This is of order 10-15 per eigenvalue found and depends upon many factors, including the accuracy required, the unpredictable effects of the FAST option described earlier, the user's initial eigenvalue estimate and how well suited the problem is to the convergence procedures described in the next section. 


\section{CONVERGENCE PROCEDURES}

VICON used the value of $J$ computed by (1) to converge on the required eigenvalues by bisection. The recent alternative "multiple determinant parabolic interpolation method" was shown to be approximately twice as fast as bisection for frame problems (Williams and Kennedy 1988). It solves the transcendental eigenvalue problem which arises when exact member theory is used to obtain the overall stiffness matrix of a structure. Therefore it was easily adapted to the VIPASA route through VICONOPT, but care was needed when using Lagrangian multipliers, i.e. when using (1) and (2), as follows.

Its key feature is that the method selects one of a list of alternative determinants specified by the program and, subject to certain conditions being met, uses parabolic interpolation through three known points on the determinant versus eigenparameter curve to predict the eigenvalue as being where the determinant is zero. A bisection step is substituted if the conditions are not met and a criterion is used to decide which determinant to use if several satisfy the conditions. The principal conditions are that the three points used bracket the required eigenvalue and no others, that the determinant plot has no poles (i.e. vertical asymptotes) between the points and that the parabola through the points is sufficiently close to the straight line through their outermost pair. The criterion picks the determinant that satisfies all the conditions and has the least relative separation between the parabola and the straight line. If $\mathbf{K}_{i}$ is temporarily thought of as the real $f N$ $\times f N$ stiffness matrix to which the method was originally applied (Williams and Kennedy 1988), the determinants used are those obtained by multiplying together the last $m$ leading diagonal elements of $\mathbf{K}_{i}^{\Delta}$ for selected values of $m$, with $m=f N$, i.e. the determinant of the complete stiffness matrix $\mathbf{K}_{i}$, usually included. Theoretical proofs guarantee that the method converges on eigenvalues without missing any and without ever taking many more iterations than bisection would. These proofs depend upon $J$ being known and upon properties of $\mathbf{K}_{i}$ that enable poles to be detected. Fortunately the proofs extend to apply when $J$ is given by (1) and the determinants are obtained by multiplying together the last $m$ elements of the array obtained by writing in sequence the leading diagonals of $\mathbf{K}_{f}^{\Delta}$, of the $\mathbf{K}_{i}^{\Delta}$, and of $\mathbf{R}^{\Delta}$, which are all calculated anyway when finding $s\left\{\mathbf{K}_{f}\right\}$, the $s\left\{\mathbf{K}_{i}\right\}$ and $s\{\mathbf{R}\}$ in (1). The extended proof has not been given anywhere, but starts from the published (Anderson et al. 1983; Williams and Anderson 1983) derivation of (1).

The value of $J$ is always even for the very common situations for which $\alpha_{f}$ and all of the $\alpha_{i}$ are equal to two, so that then eigenvalues always occur in coincident pairs. A physical interpretation is that when the number of longitudinal bays over which the mode repeats [i.e. $2 / \xi$, equals three for Fig. 2(a)] exceeds two, moving the original mode along the plate assembly by $l$ gives an alternative mode, because the two modes are distinct when viewed from a common vantage point.

The problem could have been formulated using entirely real arithmetic, resulting in real symmetric stiffness matrices whose determinant plots touch (but do not cross) the eigenparameter axis. However the complex formulation used in VICONOPT results in these matrices being replaced by Hermitian ones of half the order, such that each (real) leading diagonal element of the Hermitian $\mathbf{K}_{i}^{\Delta}$ corresponds to a pair of equal leading diagonal elements of the real $\mathbf{K}_{i}^{\Delta}$. A similar thing happens for $\mathbf{K}_{f}$ and $\mathbf{R}$. Thus the determinant of the complex formulation is equal in magnitude to the square root of that 
for the real formulation and crosses the eigenparameter axis were the latter touches it. It may therefore be used for parabolic interpolation and hence, when $\alpha_{f}$ and all of the $\alpha_{i}$ are equal to two, the condition that only one eigenvalue must be spanned by the parabola is replaced by the condition that the value of $J$ at one point must differ by two from its value at the other two points.

Computer runs showed that including this modified form of the multiple determinant parabolic interpolation method within VICONOPT typically saved over $50 \%$ of the iterations previously needed by the bisection method of VIPASA and VICON. These runs found eigenvalues to a relative accuracy of $10^{-6}$, which is approximately the accuracy needed to obtain reliable modes.

\section{FUTURE DEVELOPMENTS}

Since the release of VICONOPT in 1990, substantial further developments have begun. This section outlines additional features which are expected to appear in future releases.

The exact flat plate theory of VIPASA (Wittrick and Williams 1974) is being extended to optionally include first-order shear deformation plate theory, so giving more accurate results for panels containing thick plates. While the plate stiffness matrix is still derived by solving the governing differential equations, this new solution is obtained numerically rather than analytically.

Similar numerical solutions are also applied to cases which could not previously be solved analytically. For example, plates with completely general $\mathbf{A}, \mathbf{B}$, and $\mathbf{D}$ matrices are allowed, so removing the restriction to balanced symmetric laminates. Numerical solutions also simplify mode finding by allowing a more efficient calculation of modal displacements within plates than that described previously.

Plates with curved cross sections or tapered geometry, properties or loads could previously be handled efficiently by linking numerous flat plates in substructures. A new feature allows concise definition of the whole plate in data, with the substructuring being performed automatically, optionally allowing for transverse shear effects.

Some additional loading conditions are being introduced: bending loads causing moments or curvatures about the $y$-and $z$-axes of Fig. 1(b); pressure loading, causing bending about the $y$-axis for a number of different boundary conditions; and an initial bow in the $z$-direction causing bending about the $y$-axis of Fig. $1(b)$. These all supplement the total longitudinal load or uniform longitudinal strain in the preliminary calculation of the dead and live loads in each plate.

It is proposed to allow VICON constraints (i.e. point supports and supporting structure attachments) in substructures. This is expected to improve solution times by allowing more efficient use of substructures when modeling.

Finally, the program is being made more user friendly by the introduction of interactive procedures for data preparation, input, and editing.

\section{EXAMPLES}

The first example presented is the composite blade stiffened panel of Fig. 6, which was also used as example 1 by Stroud et al. (1984) when comparing VIPASA results with those of the finite-element programs EAL and STAGS 


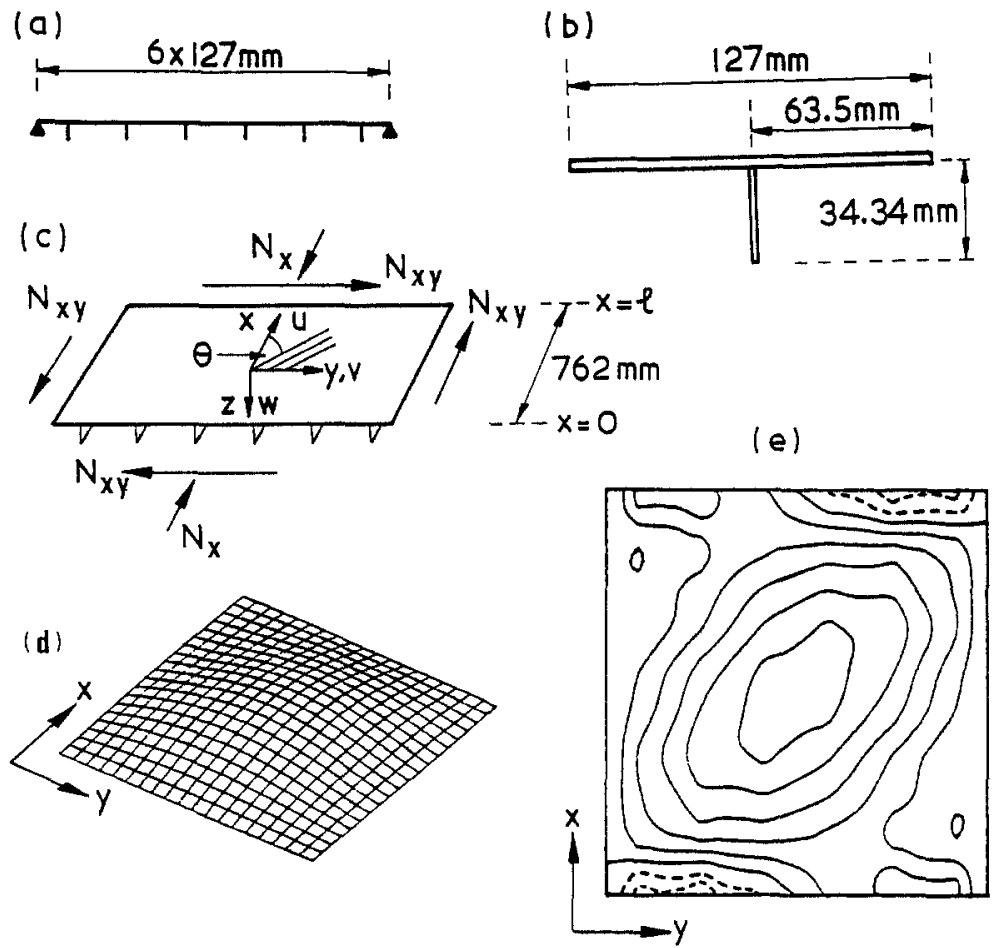

FIG. 6. Composite Panel: (a) Cross Section; (b) Repeating Portion; (c) Isometric View; (d) Isometric View of Midsurface Skin Displacements for First Result in Table 1; (e) Corresponding Contour Plot of Stress $\sigma_{x}$ at Midsurface of Upper $0^{\circ}$ Skin Ply (Negative is Dashed)

(Almroth et al. 1982). The material properties were $E_{1}=131 \mathrm{GPa}, E_{2}=$ $13.0 \mathrm{GPa} ; E_{12}=6.41 \mathrm{GPa} ; \nu_{12}=0.38$; and $\nu_{21}=0.0378$. With positive angles denoted by $\theta$ on Fig. $6(c)$, the laminates were $\left[45^{\circ},-45^{\circ},-45^{\circ}, 45^{\circ}\right.$, $\left.0^{\circ}, 90^{\circ}\right]_{s}$ for the skin and $\left[45^{\circ},-45^{\circ},-45^{\circ}, 45^{\circ}, 0^{\circ}\right]_{s}$ for the blade, with all thicknesses being $0.1397 \mathrm{~mm}$ except for the $90^{\circ}$ fibers for the skin and the $0^{\circ}$ ones for the blade, for which the thicknesses were, respectively, 1.2573 $\mathrm{mm}$ and $0.2794 \mathrm{~mm}$. The panel was simply supported on all four edges. Factored longitudinal and shear loads were applied, with the latter confined to the skin.

Table 1 gives the results, to the first of which the two VICONOPT graphical output examples of Figs. 6(d and e) relate. The load factor shown was applied to both $N_{x}$ and $N_{x y}$. The VIPASA results were obtained by Stroud et al. (1984) to demonstrate that VIPASA could be unacceptably conservative. However, discussion of why the various other results given in Table 1 differ from each other is given in the following section rather than here. For the VICONOPT results with $r=17$ or 3 the constraints prevented $w$ displacement of the skin at the blade attachments and halfway between them and also prevented $v$ displacement at the blade tips, whereas when $r$ $=12$ or 2 the $w$ constraints between blades were omitted. These constraints were all input at $x=0$ and their repetition at intervals of $l$ was implied, see Fig. 2(a). For the full analysis given in the first three rows for each of 
TABLE 1. Buckling Load Factors and Dimensionless Solution Times for Example $1\left(N_{x y}=175.13 \mathrm{kN} / \mathrm{m}\right)$

\begin{tabular}{l|c|c|c|c|c|c|c}
\hline \hline $\begin{array}{c}\text { Type of } \\
\text { analysis } \\
(1)\end{array}$ & $\begin{array}{c}N_{x} / N_{x y} \\
(2)\end{array}$ & $\begin{array}{c}r, q \\
(3)\end{array}$ & $\begin{array}{c}\text { Factor } \\
(4)\end{array}$ & $\begin{array}{c}\xi, \eta \\
(5)\end{array}$ & $\begin{array}{c}\text { Actual } \\
\text { time } \\
(\%) \\
(6)\end{array}$ & $\begin{array}{c}\text { Eigen- } \\
\text { values, } \\
\text { iterations } \\
(7)\end{array}$ & $\begin{array}{c}\text { Predicted } \\
\text { time } \\
(\%) \\
(8)\end{array}$ \\
\hline VICONOPT & 1.0 & 17,5 & 0.927 & $1.0,-$ & 2.30 & 1,18 & 2.44 \\
VICONOPT & 1.0 & 12,5 & 0.925 & $1.0,-$ & 1.43 & 1,18 & 1.38 \\
VICONOPT & 1.0 & 12,2 & 0.936 & $1.0,-$ & 0.61 & 1,16 & 0.58 \\
Repetitive & 1.0 & 3,5 & 0.820 & $0.75,0.17$ & 1.39 & 2,65 & 0.75 \\
Repetitive & 1.0 & 2,5 & 0.818 & $0.75,0.17$ & 0.77 & 2,65 & 0.41 \\
VIPASA $\lambda=l$ & 1.0 & - & 0.417 & - & 0.09 & 1,11 & 0.09 \\
EAL & 1.0 & - & 0.840 & - & - & - & - \\
STAGS coarse & 1.0 & - & 0.868 & - & 4.60 & - & - \\
STAGS medium & 1.0 & - & 0.851 & - & 29.9 & - & - \\
STAGS fine & 1.0 & - & 0.842 & - & 100 & - & - \\
VICONOPT & 0.5 & 17,5 & 1.298 & $0.75,-$ & 5.76 & 2,32 & 5.66 \\
VICONOPT & 0.5 & 12,5 & 1.285 & $0.75,-$ & 3.46 & 2,32 & 3.17 \\
VICONOPT & 0.5 & 12,2 & 1.304 & $0.75,-$ & 1.91 & 3,47 & 1.80 \\
Repetitive & 0.5 & 3,5 & 1.114 & $0.50,0.33$ & 2.04 & 5,94 & 1.06 \\
Repetitive & 0.5 & 2,5 & 1.106 & $0.50,0.33$ & 1.11 & 5,92 & 0.55 \\
VIPASA $\lambda=l$ & 0.5 & - & 0.485 & - & 0.09 & 1,10 & 0.09 \\
EAL & 0.5 & - & 1.206 & - & - & - & - \\
\hline \hline
\end{tabular}

the two values of $N_{x} / N_{x y}$, the number of VIPASA stiffness matrices coupled was $q$ when $\xi=0$ or 1 and $2 q+1$ otherwise, whereas for the repetitive analysis given in the next two rows the number was $2 q-1$ when $\xi=0$; $2 q$ when $\bar{\xi}=1$; and $2 q+1$ otherwise. (The program uses $q=5$ by default.) The values of $\xi$ considered were $1,0,0.75,0.5$, and 0.25 in that order, and the repetitive analyses were run for $\eta= \pm 1 / 6, \pm 2 / 6, \ldots, \pm 5 / 6,1$. (Note that $\eta=0$ was omitted because its only possible buckling modes are a wide column one, at a lower buckling load than for simply supported longitudinal edges, and a local one that is not relevant when finding overall modes.) The FAST option was used, and so the solution times depended largely upon how many values of $\xi$ and $\eta$ had been considered fully before the lowest eigenvalue was found. This information is given in the penultimate column of Table 1, together with the total iteration count.

The last column of Table 1 gives estimated solution times, based on the quantities $t_{1}, \ldots, t_{7}$ defined in and around (3) $-(5)$ and the actual number of iterations required. Allowance was made for overheads such as data reading and mode finding, but not for the additional plate stiffness transformations required in repetitive problems. Thus the predictions for the repetitive cases consistently underestimated the actual solution times. The results for EAL were the well converged ones (Stroud et al. 1984), for which comparative solution times were not available.

The STAGS run used rectangular element 411 (Almroth et al. 1982). The coarse mesh used 20 elements along the length of the panel and one element widthwise for each of the six blades, the five skin portions between blades and the two remaining skin portions. The medium mesh differed only because two elements were used across each of the five skin portions between blades. The fine mesh divided every element of the medium mesh into two halves widthwise. The time taken to find the buckling load for the fine mesh 
was used to make all solution times dimensionless. VICONOPT and STAGS were not run on the same computer, but the program PASCO (Anderson and Stroud 1981) was used to solve a typical problem on both machines in order to obtain a conversion factor to relate all the times in Table 1 to a common base, such that $100 \%$ in Table 1 equated approximately to 1,062 central processing unit (CPU) seconds on a VAX 6320 computer. STAGS was only run for $N_{x}=N_{x y}$, and similar times might be expected for $N_{x}=$ $0.5 N_{x y}$.

The versatility and efficiency of VICONOPT are illustrated by the second example, namely the corrugated, ring-stiffened, laminated cylinder of Fig. 7, for which the User Manual (Williams et al. 1990a) gives full numerical details. The cylinder was loaded in shear and compression, with thermal effects included. Radial and tangential skin displacements were constrained by transverse simply supported edges (represented by the point supports shown) and attachments of the inner flanges to three ring stiffeners, represented by supporting structures. The cylinder was modeled efficiently using cylindrical coordinate transformations (so only five angles needed to be given in data) and the repetitive analysis, which used only a repeating portion comprising two nodes on the outer flange, one node on the inner flange, three point supports (each preventing two displacements for each node at $x=0$ ) and three attachments to the supporting structures (each linking two displacements of the skin and ring stiffeners at $x=l / 4, l / 2$, and $3 l / 4)$. The FAST option was used to obtain the lowest buckling load over 226 combinations of $\xi$ and $\eta$, from which only six eigenvalues were found, the remainder being eliminated by check iterations. Fig. 7(c) shows a contour plot of the radial displacement around $1 / 4$ of the circumference. The contour lines are wrinkled because the inner and outer flanges had different deflection patterns, an effect made more pronounced by the ring stiffener attachments.

(a)
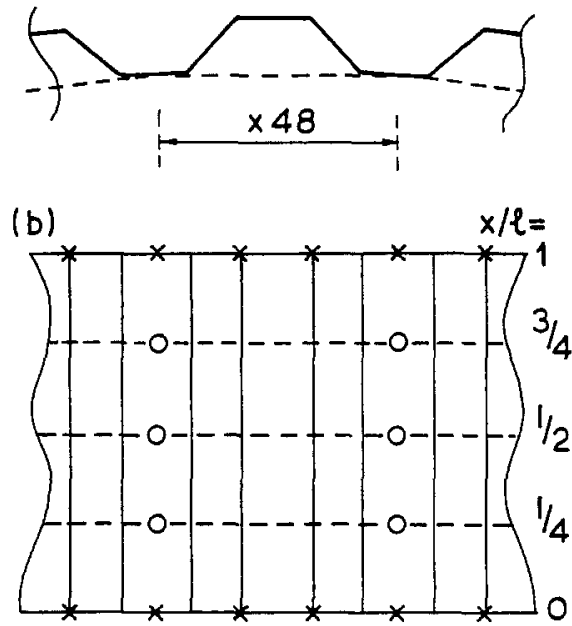

(c)

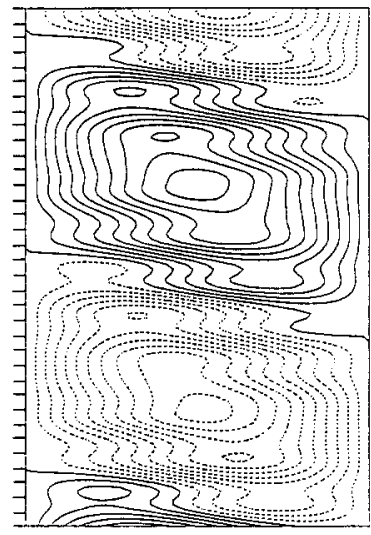

FIG. 7. Cylinder with 48 Regular Corrugations and Three Ring Stiffeners (Shown Dashed): (a) Typical Portion; (b) Side View (Foreshortened), Showing Point Supports $(x)$ and Attachments to Rings $(O)$; (c) Contour Plot of Radial Displacements of 1/4 Cylinder at Lowest Buckling Load (Negative is Dashed) 


\section{COMMENT ON RESULTS}

The STAGS results of Table 1 appear to be converging to the EAL result of 0.840 as the mesh is refined, and the results of the VICONOPT full analysis were similarly converged at 0.927 . The difference of about $10 \%$ between these results is typical for panels with high shear loading. Such differences reduce as $N_{S}$ is reduced relative to $N_{L}$ and $N_{T}$ and are zero when every plate has $D_{13}=D_{23}=N_{S}=0$. They arise because the converged STAGS results were for a panel of finite length $l$, whereas the VICONOPT results were for an infinitely long panel simply supported at longitudinal intervals of $l$ and therefore probably model more accurately the continuity with adjacent panels and other structure which exists in most practical applications, e.g. aircraft wings.

The results of Table 1 show that the medium or even the coarse STAGS mesh gave the buckling load factor sufficiently accurately for most engineering purposes. This is because overall buckling governed the response for this example. However, local buckling governs for many other panels and it is often not known in advance whether this will be the case, particularly when using optimization procedures that may converge to panels having coincident or close local and overall buckling loads. Then the mesh needs to be fine enough to predict local buckling accurately and hence the use of the fine mesh as the datum for the time comparisons in Table 1 was more than justified by the following additional results.

To check whether STAGS would need more elements to obtain sufficient accuracy when the governing mode is local, the skin portion between blades was removed from the panel and analyzed with its ends simply supported and with its longitudinal edges either simply supported or clamped. for the $N_{x}=N_{x y}$ case of Table 1 , the numbers of elements needed to get within $1 \%$ of the converged buckling load were found to be approximately 30 in the longitudinal direction, and 4 in the transverse direction for the simply supported ( 7 for clamped) case.

The full VICONOPT result of 0.936 for $r=12$ and $q=2$ given in Table 1 was sufficiently close to the converged answer of 0.927 for most engineering purposes and took well under $1 \%$ of the datum solution time. This percentage would be lower for panels with more stiffeners, because whereas VICONOPT and STAGS times are both increased by the larger number of nodes needed across the width of the panel, the STAGS runs are also likely to need more elements in the longitudinal $x$-direction, due to the higher aspect ratios of the interstiffener skin portions (compared with 6 for example 1) and hence higher ratios between their length and the half-wavelength of local buckling. In such cases VICONOPT can detect local buckling by performing VIPASA analyses at half-wavelengths in the vicinity of the width of the skin portion, with negligible increase in solution time, e.g. see the comparable VIPASA times in Table 1. Similarly, the advantage of using VICONOPT will also be greater than two orders of magnitude when using stiffeners such as hats, the width of which reduces the width of unsupported skin between them.

Solution times depend upon the order of presentation of the values of $\xi$ (and of $\eta$ for repetitive analysis) in data because the FAST option finds only critical load factors that are lower than any already found. For the full analysis with $N_{x}=N_{x y}$, the penultimate column of Table 1 shows that the first eigenvalue found was the lowest one for all three $r$ and $q$ combinations given in Table 1 and so solution was very fast. For the corresponding $N_{x}$ $=0.5 N_{x y}$ results the second or third eigenvalue found was the lowest one, 
so the solution times for this case were somewhat greater. Similarly in the repetitive analyses, the lowest eigenvalue for the $N_{x}=N_{x y}$ case was found faster than that for the $N_{x}=0.5 N_{x y}$ case.

The repetitive method can be invaluable for regular polygonal cross sections, which represent cylinders very accurately, giving the same answer as a full VICONOPT analysis. For instance, a full solution was estimated to need at least three orders of magnitude more time than was used to solve example 2 (see Fig. 7), and the equivalent finite-element calculation would be a very major undertaking. In contrast, the repetitive method gave only small time savings when applied to example 1 (see Table 1 , but also the previous paragraph) and its answer of 0.820 was not very close to the 0.927 of the full analysis, with which it should be compared because both results were for an infinitely long panel with supports that repeat at longitudinal intervals of $l$. However the method still has value for flat panels both because it gives a progressively increasing time advantage over full analysis as the number of stiffeners is increased and also because it gives lower answers and so is likely to be closer to the result for finite-length panels, e.g. compare the load factors of 0.820 and 0.840 in Table 1 .

Data preparation took several times longer for STAGS than for VICONOPT and the latter had computer memory requirements of only between 9,000 and 17,000 floating-point real numbers. In contrast the EAL and STAGS runs of Table 1 each required core storage well in excess of 100,000 floating-point numbers and also used external backing store.

\section{MAIN CONCLUSIONS}

VICONOPT finds eigenvalues (i.e. critical buckling loads or natural frequencies) and modes for any prismatic assembly of flat plates that each carry a general longitudinally invariant in-plane stress system and have anisotropic bending properties. Major features combine to make modeling of complex cross sections relatively easy and to give very fast solutions. These features include a guarantee that modes are never missed; very powerful multilevel substructuring; calculation and plotting of modes within substructures and of stresses in or between plies of laminated plates; the ability to transform to cylindrical coordinates or back to Cartesian ones at will; attachment to transverse beams or transverse plane frames; a very fast analysis of repetitive cross sections that introduces no additional approximations when applied to cylindrical structures; and a convergence method that is typically more than twice as fast as bisection.

For the usual simply supported end conditions, earlier VIPASA theory is used to obtain exact results, unless some plates are anisotropic and/or loaded in shear. Otherwise, there is the option of finding local modes by VIPASA theory and overall modes by VICON theory, which couples VIPASA responses with appropriate half-wavelengths by a Lagrangian multiplier method. Results show that this VICON method converged rapidly to the correct answer as the number of half-wavelengths, and the number of point supports used to represent the simple supports, were both increased.

VICONOPT accounts for the continuity over several bays, that typifies wing and fuselage construction, by solving for the infinitely long structure obtained by repeating the bay and its supports longitudinally. In contrast typical finite-element solutions are for a single bay with simply supported ends and so give overall buckling loads that are about $10 \%$ lower when shear loads are high.

Although care must be taken when generalizing from so few results, 
comparison with STAGS finite-element results was sufficiently reliable to show that data preparation was several times quicker and that solution times were typically two or more orders of magnitude faster, the higher orders including approximately three when VIPASA analysis was sufficient and four or more when repetitive analysis was used for cylindrical cross sections. In the absence of anisotropic and/or shear loaded plates, the VICONOPT and fully converged STAGS results would be identical for all problems with suitable arrangements of point supports.

\section{ACKNOWLEDGMENTS}

This work was sponsored by NASA under Cooperative Agreement No. NCCW-000002 and Contract No. NAS1-18584 Task No. 5, with the United Kingdom work also supported by British Aerospace.

\section{APPENDIX I. REFERENCES}

Almroth, B. O., Brogan, F. A., and Stanley, G. M. (1982). "Structural analysis of general shells, Vol. II: user instructions for STAGSC-1." Rep. LMSC-D633873, Lockheed Palo Alto Research Laboratory, Palo Alto, Calif.

Anderson, M. S., Hennessy, K. W., and Heard, W. L. Jr. (1976). "Addendum to users guide to VIPASA (vibration and instability of plate assemblies including shear and anisotropy)." NASA TM X-73914, National Aeronautics and Space Administration (NASA), Washington, D.C.

Anderson, M. S., and Stroud, W. J. (1981). "PASCO: structural panel analysis and sizing code; user's manual." NASA TM-80182, National Aeronautics and Space Administration (NASA), Washington, D.C.

Anderson, M. S., Williams, F. W., and Wright, C. J. (1983). "Buckling and vibration of any prismatic assembly of shear and compression loaded anisotropic plates with an arbitrary supporting structure." Int. J. of Mech. Sci., 25(8), 585-596.

Butler, R., and Williams, F. W. (1990). "Optimum design features of VICONOPT, an exact buckling program for prismatic assemblies of anisotropic plates." Proc., 31st AIAA/ASME/ASCE/AHS/ASC Struct., Struct. Dynamics and Mat. Conf., ASCE, New York, N.Y., 1289-1299.

Kennedy, D., and Williams, F. W. (1990). "Vibration and buckling of anisotropic plate assemblies with Winkler foundations." J. Sound and Vibration, 138(3), 501510.

Stroud, W. J., Greene, W. H., and Anderson, M. S. (1984). "Buckling loads of stiffened panels subjected to combined longitudinal compression and shear: results obtained with PASCO, EAL and STAGS computer programs." NASA TP-2215, National Aeronautics and Space Administration (NASA), Washington, D.C.

Williams, F. W. (1972). "Computation of natural frequencies and initial buckling stresses of prismatic plate assemblies." J. Sound and Vibration, 21(1), 87-106.

Williams, F. W., and Anderson, M. E. (1973). "User's guide to VIPASA (vibration and instability of plate assemblies including shear and anisotropy)." Rep., Department of Civil Engineering, University of Birmingham, Birmingham, England.

Williams, F. W., and Anderson, M. S. (1983). "Incorporation of Lagrangian multipliers into an algorithm for finding exact natural frequencies or critical buckling loads." Int. J. Mech. Sci., 25(8), 579-584.

Williams, F. W., and Anderson, M. S. (1985). "Buckling and vibration analysis of shear-loaded prismatic plate assemblies with supporting structures, utilizing symmetric or repetitive cross-sections." Aspects of the analysis of plate structures-a volume in honour of W. H. Wittrick, D. J. Dawe, R. W. Horsington, A. G. Kamtekar, and G. H. Little, eds., Oxford University Press, Oxford, England, 5171.

Williams, F. W., Anderson, M. S., Kennedy, D., Butler, R., and Aston, G. (1990a). User manual for VICONOPT; NASA CR-181966, National Aeronautics and Space Administration (NASA), Washington, D.C. 
Williams, F. W., and Kennedy, D. (1988). "Reliable use of determinants to solve non-linear structural eigenvalue problems efficiently." Int. J. for Numerical Methods in Engrg., 26(8), 1825-1841.

Williams, F. W., Kennedy, D., and Anderson, M. S. (1990b). "Analysis features of VICONOPT, an exact buckling and vibration program for prismatic assemblies of anisotropic plates." Proc., 31st AIAA/ASME/ASCE/AHS/ASC Struct., Struct. Dynamics and Mat. Conf., ASCE, New York, N.Y., 920-929.

Williams, F. W., and Wittrick, W. H. (1983). "Exact buckling and frequency calculations surveyed." J. Struct. Engrg., ASCE, 109(1), 169-187.

Williams, F. W., and Wright, C. J. (1978). "A compact computer program for calculating buckling stresses and natural frequencies of vibration of prismatic plate assemblies." Int. J. Numerical methods in Engrg., 12(9), 1429-1456.

Wittrick, W. H., and Williams, F. W. (1971). "A general algorithm for computing natural frequencies of elastic structures." Quarterly J. Mech. and Appl. Mathematics, 24(3), 263-284.

Wittrick, W. H., and Williams, F. W. (1973). "An algorithm for computing critical buckling loads of elastic structures." J. Struct. Mech., 1(4), 497-518.

Wittrick, W. H., and Williams, F. W. (1974). "Buckling and vibration of anisotropic or isotropic plate assemblies under combined loadings." Int. J. of Mech. Sci., 16(4), 209-239.

\section{APPENDIX II. NOTATION}

The following symbols are used in this paper:

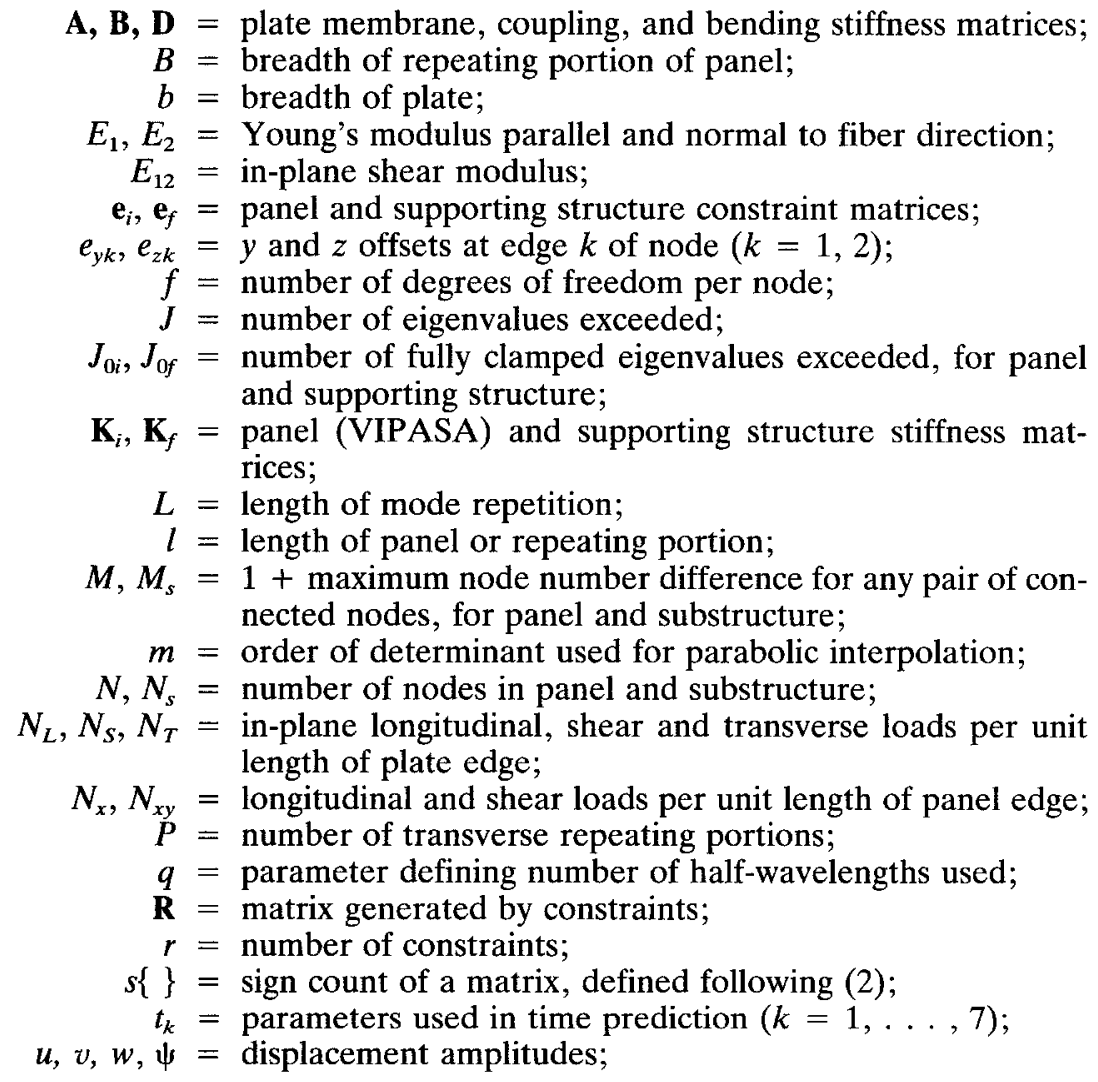




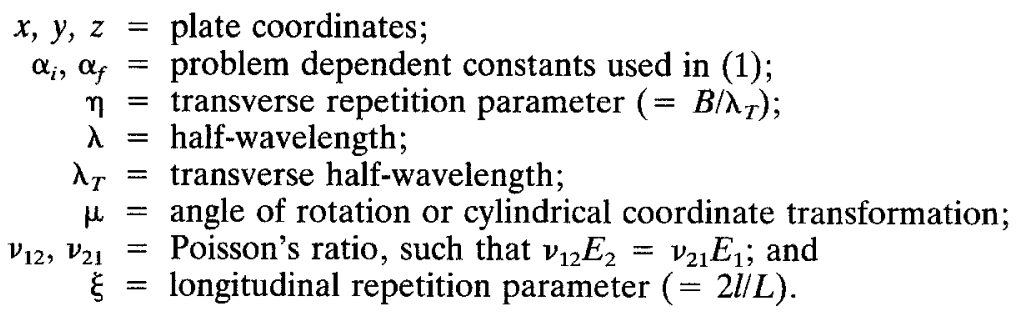

\section{Subscripts}

$$
\begin{aligned}
& f=\text { plane frame supporting structure; } \\
& i=\text { sequence number of half-wavelength used; and } \\
& s=\text { substructure. }
\end{aligned}
$$

$$
\begin{aligned}
& \text { Superscripts } \\
& \qquad \begin{aligned}
H & =\text { Hermitian transpose of matrix } \\
T & =\text { transpose of matrix; and } \\
\Delta & =\text { upper triangular form of matrix. }
\end{aligned}
\end{aligned}
$$

